\title{
Quantifying the proportion of general practice and low-acuity patients in the emergency department
}

Yusuf Nagree

MB BS, FACEM, MBA(E)

Professor of Emergency

Medicine,' and Emergenc

Physician $^{2}$

Vanessa J Camard

Research Assistant

Emergency Medicine

Research Unit ${ }^{2}$

Daniel M Fatovich

MB BS, FACEM, PhD,

Professor of Emergency

Medicine, ${ }^{1}$ and Emergency

Physician $^{3}$

Peter A Cameron

MB BS, MD, FACEM

Professor of Emergency

Medicine

Ian Dey

MB BS, FACEM

Director of Emergency

Medicine $^{2}$

Andrew D Gosbell

PhD,

Director of Policy and

Research 5

Sally M McCarthy

MB BS, FACEM, MBA

Medical Director

David Mountain

MB BS, FACEM,

Academic Emergency

Physician,' and Head o Emergency $^{7}$

1 School of Primary Aboriginal and Rural Health Care, University of Western Australia, Perth, WA.

2 Fremantle Hospital, Fremantle, WA

3 Royal Perth Hospital, Perth, WA.

4 School of Public Health. Monash University,

Melbourne, VIC

5 Australasian College for

Emergency Medicine

Melbourne, VIC

6 Emergency Care Institute, NSW Agency for Clinica Innovation, Sydney, NSW.

7 Sir Charles Gairdne Hospital, Perth, WA.

yusuf.nagree@

health.wa.gov.au

MJA 2013; 198: 612-615 doi: 10.5694/mjal2.11754

Editorial p 573
B etween June 2005 and October 2009, attendances to emergency departments (EDs) across Australia increased by $21.2 \%$. This represents an annual increase of $4.9 \%$, which significantly exceeds the rate of population growth. ${ }^{1}$ Reasons postulated include an ageing population, the rising incidence of chronic illness and decreased availability of general practitioners, especially for after-hours and urgent visits. The latter issue has led to suggestions that overcrowding in EDs is due to patients with general practice-type conditions attending inappropriately and is driven to some extent by poorly validated definitions of "inappropriate" attendances. ${ }^{2}$

In Australia, methods to determine the number of general practice-type patients attending EDs often use the Australasian Triage Scale (ATS). The ATS is a method used to prioritise care within EDs, with all patients allocated a category from 1 to 5 on arrival. Category 1 patients are regarded as the highest priority, while category 5 patients are the least urgent. ${ }^{3}$ It has been postulated that general practicetype patients are associated with ATS categories 4 and 5, but this is not well founded. $1,3-5$

The ATS is an urgency scale, not a complexity scale. A patient can have a low triage category but need complex care. An example is an elderly patient living independently who falls and fractures her forearm. She is low in urgency but high in complexity, requiring extended allied health support to ensure safe discharge. Such a patient cannot be easily managed in most GP settings. Conversely, some highurgency patients are low complexity. A young patient with fever and a rash should be seen rapidly to assess for serious illnesses such as meningitis. However, once serious illness is excluded, the patient can usually be

Abstract

Objective: To accurately estimate the proportion of patients presenting to the emergency department (ED) who may have been suitable to be seen in general practice.

Design: Using data sourced from the Emergency Department Information Systems for the calendar years 2009 to 2011 at three major tertiary hospitals in Perth, Western Australia, we compared four methods for calculating general practice-type patients. These were the validated Sprivulis method, the widely used Australasian College for Emergency Medicine method, a discharge diagnosis method developed by the Tasmanian Department of Human and Health Services, and the Australian Institute of Health and Welfare (AlHW) method.

Main outcome measure: General practice-type patient attendances to EDs, estimated using the four methods.

Results: All methods except the AlHW method showed that $10 \%-12 \%$ of patients attending tertiary EDs in Perth may have been suitable for general practice. These attendances comprised 3\%-5\% of total ED length of stay. The AlHW method produced different results (general practice-type patients accounted for about 25\% of attendances, comprising 10\%-11\% of total ED length of stay). General practice-type patient attendances were not evenly distributed across the week, with proportionally more patients presenting during weekday daytime (08:00-17:00) and proportionally fewer overnight (00:00-08:00). This suggests that it is not a lack of general practitioners that drives patients to the ED, as weekday working hours are the time of greatest GP availability.

Conclusion: The estimated proportion of general practice-type patients attending the EDs of Perth's major hospitals is $10 \%-12 \%$, and this accounts for $<5 \%$ of the total ED length of stay. The AlHW methodology overestimates the actual proportion of general practice-type patient attendances.

discharged. This patient might be equally well managed in a primary care environment by experienced GPS.

Estimating the proportion of ED attendees suitable for general practice is complex, with experienced researchers devising a number of approaches to quantify such patients. There is no agreed standard definition for identifying patients as appropriate or inappropriate to attend an ED. A recent extensive literature review ${ }^{4}$ found that the calculated rate of non-urgent ED attendances varied between $4.8 \%$ and $90 \%$, confirming that there is no standard methodology for determining the true proportion of general practicetype patients in EDs.

A number of methods are currently used in Australasia to estimate the number of general practice-type patients presenting to EDs. ${ }^{1,5,6}$ Only one of these has been validated in the literature. ${ }^{6}$ We aimed to compare this validated method with other Australasian methods using data from three major tertiary hospitals in Perth, Western Australia.

\section{Methods}

Data were extracted from the Emergency Department Information Systems (EDIS version 9.46, iSoft) for the calendar years 2009, 2010 and 2011 at three tertiary hospitals (two adult-only, one mixed) in Perth. Average annual census was 55000, with admission rates between $35 \%$ and $55 \%$.

Four methods were used to estimate the number of general practice-type patients:

- The Australian Institute of Health and Welfare (AIHW) method, which 


\begin{tabular}{|c|c|c|c|c|c|c|}
\hline Year & Total male and female & Male & Weekday daytime* & Weekday evening ${ }^{\dagger}$ & Overnight ${ }^{\ddagger}$ & Weekend $^{\S}$ \\
\hline 2009 & 164352 & $88777(54.0 \%)$ & $58595(35.7 \%)$ & $38203(23.2 \%)$ & $28726(17.5 \%)$ & $38828(23.6 \%)$ \\
\hline 2010 & 175721 & 94910 (54.0\%) & 63366 (36.1\%) & $40844(23.2 \%)$ & $30192(17.2 \%)$ & 41319 (23.5\%) \\
\hline 2011 & 192056 & $103320(53.8 \%)$ & 69719 (36.3\%) & $45085(23.5 \%)$ & $31844(16.6 \%)$ & $45408(23.6 \%)$ \\
\hline
\end{tabular}

* Mon-Fri, 08:00-17:00. † Mon-Fri, 17:00-00:00. ¥ Mon-Sun, 00:00-08:00. § Sat-Sun, 08:00-00:00.

\begin{tabular}{|c|c|c|c|c|c|c|c|c|c|}
\hline \multirow[b]{2}{*}{ Year } & \multirow[b]{2}{*}{ Total } & \multicolumn{2}{|c|}{ ACEM } & \multicolumn{2}{|c|}{ Sprivulis ${ }^{6}$} & \multicolumn{2}{|c|}{ Diagnosis* } & \multicolumn{2}{|c|}{ AlHW } \\
\hline & & No. & $95 \% \mathrm{Cl}$ & No. & $95 \% \mathrm{Cl}$ & No. & $95 \% \mathrm{Cl}$ & No. & $95 \% \mathrm{Cl}$ \\
\hline 2009 & $164352^{\dagger}$ & 16905 (10.6\%) & $10.3 \%-10.6 \%$ & $15984(9.7 \%)$ & $9.5 \%-10.0 \%$ & $17888(11.1 \%)$ & $10.9 \%-11.2 \%$ & $43452(26.4 \%)$ & $26.2 \%-26.7 \%$ \\
\hline 2010 & $175721^{\ddagger}$ & 19140 (11.1\%) & $10.9 \%-11.3 \%$ & 15741 (9.0\%) & $8.7 \%-9.2 \%$ & 19127 (11.0\%) & $10.8 \%-11.3 \%$ & $44495(25.3 \%)$ & $25.1 \%-25.6 \%$ \\
\hline 2011 & $192056^{5}$ & 21877 (11.6\%) & $11.3 \%-11.8 \%$ & 18151 (9.5\%) & $9.2 \%-9.7 \%$ & 21242 (11.2\%) & $11.0 \%-11.4 \%$ & 48041 (25.0\%) & $24.8 \%-25.2 \%$ \\
\hline
\end{tabular}

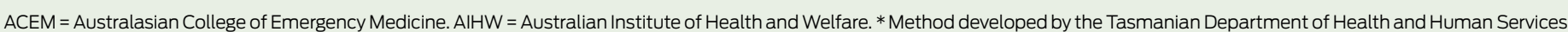

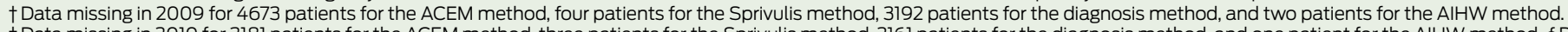

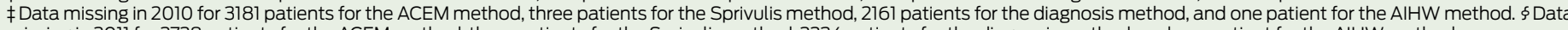

missing in 2011 for 2728 patients for the ACEM method, three patients for the Sprivulis method, 2234 patients for the diagnosis method, and one patient for the AlHW method.

considers a general practice-type patient to be any patient allocated an ATS category 4 or 5 , who does not arrive by ambulance, police or correctional vehicle, and is not admitted to hospital, is not referred to another hospital and does not die. ${ }^{1}$

- The method developed by Sprivulis, ${ }^{6}$ which examines the difference between the discharge rates of selfreferred patients and GP-referred patients, with calculations based on the self-referred, non-admitted, ATS category 3, 4 and 5 patients.

- The Australasian College for Emergency Medicine (ACEM) method, which considers that any self-referred, non-ambulance patient with a medical consultation time under 1 hour may have been suitable for a GP. ${ }^{5}$ Patients who did not wait or had an invalid consultation time are excluded as their consultation time cannot be calculated. - The diagnosis method, which uses a list of diagnoses of conditions possibly suitable for GP management. Selfreferred, non-admitted, ATS category 4 and 5 patients arriving by private transport and meeting one of these diagnoses were considered to be potential GP cases. Patients without a diagnosis were excluded as a diagnosis is required to determine if they were general practice-type. This method was originally developed by Kevin Ratcliffe at the Tasmanian Department of Health and Human Services.

Two of the hospitals have co-located after-hours GP practices, and patients presenting to the ED for a referral to these practices were excluded. These comprised less than $700(0.2 \%)$ patients over the 3 years of the study.
The WA South Metropolitan Health Service Human Research Ethics Committee considered this to be an audit activity and approval was given by the Clinical Governance Unit of Fremantle Hospital. As the data was de-identified, permission was sought and granted by the Head of ED at each hospital as per the WA Guidelines for the release of data (August 2012) and the Information access and disclosure policy (February 2012). Permission to use the diagnosis list was granted by the Tasmanian Department of Health and Human Services.

As it has been postulated by Australian governments ${ }^{7}$ that the number of general practice-type cases is greater in the evening and overnight due to lack of after-hours GPs, presentation times were grouped as overnight (00:00-08:00), weekends (Saturday and Sunday, 08:00-00:00), weekday daytime (Monday-Friday, 08:00-17:00) and weekday evening (Monday-Friday, 17:00-00:00).

\section{Results}

From 2009 to 2011, there were 532129 ED presentations (Box 1). There was a slight predominance of males, and $36.0 \%$ of attendances occurred during the weekday daytime.

Incomplete data resulted in the exclusion of 10582 (2.0\%) records from the ACEM method, 7587 (1.4\%) from the diagnosis method, four from the AIHW method and 10 from the Sprivulis method.
The ACEM, diagnosis and Sprivulis methods estimated that $10 \%-12 \%$ of patients were general practice-type, whereas the AIHW method estimated $>25 \%$ (Box 2). The confidence intervals showed some differences between the non-AIHW methods, but all ranged between $8.7 \%$ and $11.6 \%$, whereas those for the AIHW method ranged between $24.8 \%$ and $26.7 \%$.

Using the ACEM and AIHW methods, there were slightly more males in the general practice-type population compared with the total population, but the diagnosis and Sprivulis methods showed similar numbers (Box 3). Proportionally more general practicetype patients presented during the weekday daytime (Box 4). The total ED time of general practice-type patients was $3 \%-5 \%$ for the ACEM, Sprivulis and diagnosis methods and 10\%-11\% for the AIHW method (Box 5).

\section{Discussion}

Our data demonstrate that $10 \%-12 \%$ of patients attending tertiary EDs in Perth between 2009 and 2011 may have been suitable for general practice. These patients contributed $3 \%-5 \%$ of overall ED length of stay, and probably a lesser proportion of resource, staffing and cubicle usage. These data are based on consistent estimates using the Sprivulis method, which is validated in the literature, the ACEM method, which is used by the peak body for emergency medicine in Australasia, and the diagnosis method. 
3 Number of males presenting to emergency departments with general practice-type conditions, calculated by four commonly used methods

\begin{tabular}{|c|c|c|c|c|c|c|c|c|}
\hline \multirow[b]{2}{*}{ Year } & \multicolumn{2}{|c|}{ ACEM } & \multicolumn{2}{|c|}{ Sprivulis $^{6}$} & \multicolumn{2}{|c|}{ Diagnosis* } & \multicolumn{2}{|c|}{ AlHW } \\
\hline & $\begin{array}{l}\text { Total male } \\
\text { and female }\end{array}$ & Male & $\begin{array}{l}\text { Total male } \\
\text { and female }\end{array}$ & Male & $\begin{array}{l}\text { Total male } \\
\text { and female }\end{array}$ & Male & $\begin{array}{l}\text { Total male } \\
\text { and female }\end{array}$ & Male \\
\hline 2009 & 16905 & $10231(60.5 \%)$ & 15984 & 8923 (55.8\%) & 17888 & $9488(53.0 \%)$ & 43452 & $25329(58.3 \%)$ \\
\hline 2010 & 19140 & 11438 (59.8\%) & 15741 & 8776 (55.8\%) & 19127 & 10109 (52.9\%) & 44495 & $25787(58.0 \%)$ \\
\hline 2011 & 21877 & 13108 (59.9\%) & 18151 & $10164(56.0 \%)$ & 21242 & 11164 (52.6\%) & 48041 & 27855 (58.0\%) \\
\hline
\end{tabular}

ACEM = Australasian College of Emergency Medicine. AlHW = Australian Institute of Health and Welfare. * Method developed by the Tasmanian Department of Health and Human Services.

\section{Number of patients presenting with general practice-type conditions to emergency departments, calculated by four commonly used methods, by time of presentation}

General practice-type patients

\begin{tabular}{|c|c|c|c|c|c|}
\hline Time of presentation & $\begin{array}{l}\text { All patients } \\
(n=532129)\end{array}$ & $\begin{array}{c}\text { ACEM } \\
(n=57922)\end{array}$ & $\begin{array}{c}\text { Sprivulis }^{6} \\
(n=49399)\end{array}$ & $\begin{array}{l}\text { Diagnosis* } \\
(n=58257)\end{array}$ & $\begin{array}{c}\text { AlHW } \\
(n=135988)\end{array}$ \\
\hline $\begin{array}{l}\text { Weekday daytime: } \\
\text { Mon-Fri, 08:00-17:00 }\end{array}$ & $191680(36.0 \%)$ & 21135 (36.5\%) & $17399(35.2 \%)$ & 21961 (37.7\%) & $52500(38.6 \%)$ \\
\hline $\begin{array}{l}\text { Weekday evening: } \\
\text { Mon-Fri, 17:00-00:00 }\end{array}$ & 124132 (23.3\%) & $12526(21.6 \%)$ & 11026 (22.3\%) & 11497 (19.7\%) & 28268 (20.8\%) \\
\hline $\begin{array}{l}\text { Overnight: } \\
\text { Mon-Sun, 00:00-08:00 }\end{array}$ & $90762(17.1 \%)$ & $8520(14.7 \%)$ & $7862(15.9 \%)$ & $9434(16.2 \%)$ & $19560(14.4 \%)$ \\
\hline $\begin{array}{l}\text { Weekend: } \\
\text { Sat-Sun, 08:00-00:00 }\end{array}$ & 125555 (23.6\%) & $15741(27.2 \%)$ & $13112(26.5 \%)$ & $15365(26.4 \%)$ & $35660(26.2 \%)$ \\
\hline
\end{tabular}

5 Length of stay of general practice-type patients in emergency departments, as a proportion of total emergency department length of stay, calculated by four commonly used methods

\begin{tabular}{lrrrr} 
Year & ACEM & Sprivulis $^{6}$ & Diagnosis* $^{*}$ & AlHW \\
\hline 2009 & $3.1 \%$ & $5.0 \%$ & $5.3 \%$ & $11.8 \%$ \\
2010 & $2.8 \%$ & $3.8 \%$ & $4.5 \%$ & $10.1 \%$ \\
2011 & $3.3 \%$ & $4.3 \%$ & $4.9 \%$ & $10.5 \%$ \\
\hline
\end{tabular}

ACEM = Australasian College of Emergency Medicine. AlHW = Australian Institute of Health and Welfare. * Method developed by the Tasmanian Department of Health and Human Services.

A recent review in the United States concluded that $7 \%-10 \%$ of patients attending EDs may have been suitable for general practice. ${ }^{8}$ The US study used yet another method based on presenting symptom and diagnoses. However, its results were similar to those of all the Australasian methods except that of the AIHW

We found that the AIHW method consistently overestimated the proportion of general practice-type patients in EDs. We believe that this was due to its use of the ATS (an urgency rather than a complexity scale); it therefore fails to take into account the nature of the presenting condition(s), diagnostic requirements or treatment pathways. Additionally, the AIHW method includes as general practice-type, patients who have actually been referred to the ED by a GP. Admission as a proxy for complexity is becoming less relevant with admission-avoidance strategies such as home nursing. ${ }^{9}$ Complex patients can often be discharged, limiting the use of admission as a marker for complexity.

The diagnosis method might appear to be more robust as it relies on a diagnosis classification. However, using diagnoses to determine whether a specific patient needed to use an ED may be inaccurate, because triaging clinicians do not have the full clinical picture until after a full physician assessment. For example, a 70-yearold man may be discharged with a diagnosis of non-specific abdominal pain but, to reach that diagnosis, will have required an extensive workup including pathology, imaging and/or surgical consultation. A more robust method might use presenting prob- lems, as these drive the ED resource use.

The Sprivulis method is a relatively complex analytical approach that produces results consistent with those of the ACEM and diagnosis methods and the recent US study. ${ }^{8}$

The ACEM method has the advantage of being simple to calculate and intuitive. However, by assuming that a patient may be suitable for a GP if the consultation time in the ED is less than 1 hour, it may overestimate the number of general practice-type patients in the ED, as most GP consultations last about 15 minutes. Although medical consultation times in teaching hospitals can be longer than those in non-tertiary hospitals, it may be more appropriate to assume a consultation time nearer to that of general practice when extending the analysis to non-tertiary hospitals.

Reasons for patients attending the ED instead of a general practice include financial constraints and availability of access. ${ }^{10}$ Patients may also believe that their condition is an emergency. ${ }^{11,12}$ This is supported by a study that found that self-referred patients had significantly higher acuity profiles than GP-referred or healthdirectreferred patients. ${ }^{13}$ However, the rea- 
sons for general practice-type patients attending EDs are difficult to interpret because the capability of general practices can differ widely, even within a city. General practice models can range from solo practitioners with no allied health support, to practices operating in a financial environment that requires high throughput, and to "super clinics" with onsite x-ray, multidisciplinary teams and practice nurses.

Australia's dual health system funding model may provide incentives for state governments to support overestimations of the proportion of general practice-type patients attending EDs. ${ }^{7}$

After-hours GP clinics, super clinics and polyclinics may fill gaps in medical services but have minimal effects on ED attendances. 9,14-19 The impact on the ED from diverting general practicetype patients is low, and inaccurate reporting of the true proportion of these patients results in policy and program initiatives that do not address the real cause of ED overcrowding, which is the lack of available inpatient beds. ${ }^{20}$

Evidence consistently demonstrates that overcrowding leads to increased patient mortality, morbidity and prolonged hospital stays. ${ }^{14,21-23}$ Much work has been directed into trying to reduce emergency demand as a solution to reducing overcrowding; however, very few of the strategies have produced results. ${ }^{24}$

While general practice-type patients may add to waiting room numbers, they do not cause ED overcrowding or ambulance diversion and have little effect on ED workload or waiting times. ${ }^{6,14,16,17,22}$

It is also unlikely general practicetype patients will ever be completely removed from the patient cohort presenting to EDs. Small numbers of general practice-type patients will continue to present overnight, as alternative facilities do not exist and the ED provides a cost-effective model for overnight acute care as the marginal cost is minimal.

It is commonly held that the number of general practice-type cases is greater outside working hours due to a lack of after-hours GPs. However, Box 4 suggests that this is not the case. Although the weekday daytime only accounts for $27 \%$ of the week, $36 \%$ of general practice-type patients attended during this time.
It is essential to estimate accurately the proportion of general practice-type patients in the ED, as incorrect data lead to poor policy and planning. This results in misdirected and costly interventions, which inevitably fail to resolve ED overcrowding or its underlying causes. ${ }^{15,25}$

Our study was limited to three tertiary hospitals in Perth. It is likely that these results are generalisable; however, detailed analysis of the Perth population and Perth GP availability compared with the rest of Australia was beyond the scope of the study.

The proportion of general practicetype patients in outer metropolitan and regional centres may be higher; however, it is likely that overestimation by the AIHW methodology for these EDs would be of a similar magnitude. Further work is underway to extend the analysis to all WA hospitals.

The methods used here are statistical methods and will fail to capture individual patient decisions as a qualitative study might. A qualitative study is resource-intensive and impractical to conduct beyond small numbers.

The ACEM method was the easiest to use and has clinician validity for calculating the small proportion of possible general practice-type patients to EDs in Australasia, although it may require minor modifications for use outside tertiary hospitals. The AIHW methodology overestimated general practice-type patient workload in EDs and should no longer be used to guide policy decisions.

Competing interests: Yusuf Nagree, Sally McCarthy and David Mountain are councillors of the ACEM.

Received 29 Nov 2012, accepted 2 May 2013.

1 Australian Institute of Health and Welfare. Australian hospital statistics 2009-10. emergency department care and elective surgery waiting times. Canberra: AlHW, 2010. (AlHW Cat. No. HSE 93; Health Services Series No. 38.) http:// www.aihw.gov.au/publication-detail/?id= 6442472405 (accessed May 2013).

2 Richardson DB, Mountain D. Myths versus facts in emergency department overcrowding and hospital access block. Med J Aust 2009; 190: 369-374.

3 Australasian College for Emergency Medicine. Policy on the Australasian Triage Scale. 2000. http:// acem.org.au/media/policies_and_guidelines/P06_ Aust_Triage_Scale_-_Nov_2000.pdf (accessed Jan 2011).

4 Durand AC, Gentile S, Devictor B, et al. ED patients: how nonurgent are they? Systematic review of the emergency medicine literature. Am J Emerg Med 2011; 29: 333-345.

5 Australasian College for Emergency Medicine. Fact sheet re: urban emergency services - ATS 4 and 5 patients. 2001. http://www.acem.org.au/media/ ats_4_5_factsheet.pdf (accessed Jan 2011).
6 Sprivulis P. Estimation of the general practice workload of a metropolitan teaching hospital emergency department. Emerg Med (Fremantle) 2003; 15: 32-37.

7 Roxon N, D'ath Y. Work kicks off at Redcliffe GP Super Clinic [media release]. 7 May 2010. http://www.health.gov.au/internet/ministers/ publishing.nsf/Content/mr-yrl0-nr-nr086.htm (accessed Jan 2011).

8 Sommers AS, Boukus ER, Carrier E. Dispelling myths about emergency department use: majority of Medicaid visits are for urgent or more serious symptoms. Res Brief 2012; 23: 1-10, 1-3.

9 Cooke M, Fisher J, Dale J, et al. Reducing attendances and waits in emergency departments: a systematic review of present innovations. Report to the NHS Service Delivery and Organisation R\&D Programme. London: SDO, 2004.

10 Abernethie L, Nagree Y. Increased emergency department attendance: a qualitative investigation of health seeking behaviours. Just Policy 2004; 33: 6-16.

11 Singh S. Self referral to accident and emergency department: patients' perceptions. BMJ 1988; 297: $1179-1180$.

12 Masso M, Bezzina AJ, Siminski P, et al. Why patients attend emergency departments for conditions potentially appropriate for primary care: reasons given by patients and clinicians differ. Emerg Med Australas 2007; 19: 333-340.

$13 \mathrm{Ng} \mathrm{JY,Fatovich} \mathrm{DM,} \mathrm{Turner} \mathrm{VF,} \mathrm{et} \mathrm{al.} \mathrm{Appropriateness}$ of healthdirect referrals to the emergency department compared with self-referrals and GP referrals. Med J Aust 2012; 197: 498-502.

14 Fatovich DM, Nagree Y, Sprivulis P. Access block causes emergency department overcrowding and ambulance diversion in Perth, Western Australia. Emerg Med J 2005; 22: 351-354.

15 Heyworth JR. Darzi centres. Flawed assumptions have hampered emergency care. BMJ 2010; 341: c6852.

16 Nagree Y, Ercleve TN, Sprivulis PC. After-hours general practice clinics are unlikely to reduce low acuity patient attendances to metropolitan Perth emergency departments. Aust Health Rev 2004; 28: 285-291.

17 Schull MJ, Kiss A, Szalai JP. The effect of lowcomplexity patients on emergency department waiting times. Ann Emerg Med 2007; 49: 257-264, 264 el.

18 Buckley DJ, Curtis PW, McGirr JG. The effect of a general practice after-hours clinic on emergency department presentations: a regression time series analysis. Med J Aust 2010; 192: 448-451.

19 Richardson DB. Primary care services and emergency medicine [editorial]. Med J Aust 2010; 192: 429-430.

20 Fatovich DM, Hughes G, McCarthy SM. Access block it's all about available beds. Med J Aust 2009; 190: 362-363.

21 Richardson DB. Increase in patient mortality at 10 days associated with emergency department overcrowding. Med J Aust 2006; 184: 213-216.

22 Sprivulis PC, Da Silva JA, Jacobs IG, et al. The association between hospital overcrowding and mortality among patients admitted via Western Australian emergency departments. Med J Aust 2006; 184: 208-212.

23 Chalfin DB, Trzeciak S, Likourezos A, et al;DELAY-ED study group. Impact of delayed transfer of critically ill patients from the emergency department to the intensive care unit. Crit Care Med 2007; 35: 1477-1483.

24 Hoot NR, Aronsky D. Systematic review of emergency department crowding: causes, effects, and solutions. Ann Emerg Med 2008; 52: 126-136.

25 Nagree Y, Mountain D, Cameron P, et al. Determining the true burden of general practice patients in the emergency department: the need for robust methodology. Emerg Med Australas 2011; 23: $116-119$. 